\title{
Mining of haplotype-based expressed sequence tag single nucleotide polymorphisms in citrus
}

\author{
Chunxian Chen ${ }^{1,2^{*}}$ and Fred G Gmitter $\mathrm{Jr}^{1}$
}

\begin{abstract}
Background: Single nucleotide polymorphisms (SNPs), the most abundant variations in a genome, have been widely used in various studies. Detection and characterization of citrus haplotype-based expressed sequence tag (EST) SNPs will greatly facilitate further utilization of these gene-based resources.

Results: In this paper, haplotype-based SNPs were mined out of publicly available citrus expressed sequence tags (ESTs) from different citrus cultivars (genotypes) individually and collectively for comparison. There were a total of 567,297 ESTs belonging to 27 cultivars in varying numbers and consequentially yielding different numbers of haplotype-based quality SNPs. Sweet orange (SO) had the most $(213,830)$ ESTs, generating 11,182 quality SNPs in 3,327 out of 4,228 usable contigs. Summed from all the individually mining results, a total of 25,417 quality SNPs were discovered - 15,010 (59.1\%) were transitions (AG and CT), 9,114 (35.9\%) were transversions (AC, GT, CG, and AT), and 1,293 (5.0\%) were insertion/deletions (indels). A vast majority of SNP-containing contigs consisted of only 2 haplotypes, as expected, but the percentages of 2 haplotype contigs varied widely in these citrus cultivars. BLAST of the 25,417 25-mer SNP oligos to the Clementine reference genome scaffolds revealed 2,947 SNPs had "no hits found", 19,943 had 1 unique hit / alignment, 1,571 had one hit and 2+ alignments per hit, and 956 had 2+ hits and 1+ alignment per hit. Of the total 24,293 scaffold hits, 23,955 (98.6\%) were on the main scaffolds 1 to 9, and only 338 were on 87 minor scaffolds. Most alignments had 100\% (25/25) or 96\% (24/25) nucleotide identities, accounting for 93\% of all the alignments. Considering almost all the nucleotide discrepancies in the 24/25 alignments were at the SNP sites, it served well as in silico validation of these SNPs, in addition to and consistent with the rate (81\%) validated by sequencing and SNaPshot assay.

Conclusions: High-quality EST-SNPs from different citrus genotypes were detected, and compared to estimate the heterozygosity of each genome. All the SNP oligo sequences were aligned with the Clementine citrus genome to determine their distribution and uniqueness and for in silico validation, in addition to SNaPshot and sequencing validation of selected SNPs.
\end{abstract}

Keywords: Haplotype, Heterozygosity, Polymorphism, Transition, Transversion, Insertion/deletion, Non-synonymous, Synonymous

\section{Background}

Single nucleotide polymorphism (SNP) refers to an allelic single-base variation between two haplotype sequences in an individual or between any paired homologous chromosomes across homogenous members. SNPs are most abundant among genomic DNA variations and ubiquitous in both functional genes and non-coding regions [1].

\footnotetext{
* Correspondence: chunxian.chen@ars.usda.gov

'University of Florida, IFAS, Citrus Research and Education Center, 700 Experiment Station Road, Lake Alfred, FL 33850, USA

2USDA, ARS, Southeastern Fruit and Tree Nut Research Laboratory,

21 Dunbar Road, Byron, GA 31008, USA
}

Because they are conserved during evolution, associated with genetic traits, and suited for high throughput genotyping, SNPs are a popular and powerful tool for various genetics and genomics studies, such as mapping of whole genomes, tagging of important traits, comparison of genome evolution, classification of diverse clades, and many rapidly developing areas such as pharmacogenomics and functional proteomics [2-4]. These SNPs from expressed sequence tags (ESTs) represent hundreds of thousands of functional genes and likely control many genetic traits [5-8]. Due to degeneracy of most three-nucleotide genetic codons, a SNP in the coding regions may be synonymous

\section{Biomed Central}

(c) 2013 Chen and Gmitter; licensee BioMed Central Ltd. This is an open access article distributed under the terms of the Creative Commons Attribution License (http://creativecommons.org/licenses/by/2.0), which permits unrestricted use, distribution, and reproduction in any medium, provided the original work is properly cited. 
(sSNP) if it does not result in change of the protein sequence or non-synonymous (nsSNP) if it does. The nsSNPs are usually more biologically relevant because the resulting amino acid changes in proteins may change their secondary structures and functions and cause phenotypic mutations $[1,8,9]$.

SNP discovery usually is accomplished through computational alignment of redundant DNA sequences with each other or with a high-quality reference genome where discrepant nucleotides can be detected and evaluated. For the redundancy-based computational approach, in addition to sequencing errors as a source of false SNPs $[5,7,10]$, it may be even more challenging to distinguish real SNPs among allelic sequences from single nucleotide discrepancies among highly identical paralogous sequences $[8,11]$. Several bioinformatics programs (pipelines) have been developed for automatic SNP mining, using different input data, computational algorithms, quality evaluation strategies, and/or output formats. For example, the PolyPhred and PolyBayes pipeline typically requires sequence trace files or extracted sequences with base calling quality values to minimize false SNPs resulting from sequencing errors [12-14]. PolyBayes also includes an extra implementation to identify paralogs and their derived false SNPs [13]. Others like autoSNP and QuailitySNP can accept sequences without quality files for initial redundancy-based detection, and then grade SNPs by confidence levels, which are more commonly used with public ESTs that usually do not have trace or quality files $[8,15]$. The QualitySNP pipeline implements a haplotype reconstruction algorithm and confidence scoring approach to detect reliable synonymous and non-synonymous SNPs from public ESTs without quality files and a reference genome [8]. In other words, it reclusters ESTs in a contig to determine the potential haplotypes in the contig. Only single discrepant nucleotides between any two reconstructed haplotypes would be scored a potential SNP. Sequencing differences can also result from sequencing errors or alignment of paralogs. Only those potential SNPs passing additional confidence interrogation are identified as quality SNPs. Reliable quality SNPs represent the different alleles (haplotypes) of a gene. As opposed to low-confidence and false SNPs, the use of quality SNPs can benefit allele-trait association studies [8].

Most citrus species are diploid $(2 \mathrm{n}=2 \times=18)$, with highly heterozygous and relatively small genomes and over 30,000 predicted genes [16]. In general, citrus refers to true biological species and ancestrally domesticated introgressions in Citrus and those in the sexually compatible Fortunella (kumquat) and Poncirus (trifoliate orange) genera. Citrus fruit types are diverse, and include sweet orange (Citrus sinensis), mandarin (C. reticulata), grapefruit (C. paradisi), lemon ( $C$. limon), lime
(C. aurantifolia), pummelo (C. maxima), and citron (C. medica). Each type consists of many cultivars primarily selected from spontaneous bud sports, chance seedlings, induced mutants, or conventional hybrids. It is widely believed that only C. maxima, $C$. reticulata, and $C$. medica are true species, although the binomial names for the other ancestral hybrid and introgression cultivars are widely accepted and used $[17,18]$. These citrus types likely vary in levels of heterozygosity and share alleles resulting from early introgressions across these genomes, according to SSR markers [19-21]. A haploid Clementine genome sequence was produced using Sanger technology, and one diploid sweet orange genome using Roche 454 technology [22], along many other citrus genomes using other re-sequencing platforms (Gmitter et al. unpublished data). Together with other available citrus genomic resources, it is now possible for SNP detection and comparison of large-volume citrus Sanger EST datasets within and among different citrus cultivars. These gene-based SNPs, once available for the citrus community, will be very valuable in many genetic and genomic studies, and helpful for trait-targeted breeding as well $[20,21,23]$.

In this paper, SNPs in public ESTs from 27 different citrus genotypes were detected by the QualitySNP pipeline and compared to estimate the heterozygosity of each genome. All of the short SNP oligo sequences were also aligned with the Clementine citrus genome to determine their distribution and uniqueness in the genome and for in silico validation. Selected SNPs were also validated by SNaPshot and sequencing.

\section{Methods}

\section{Citrus ESTs and cultivars}

All citrus ESTs were retrieved from the National Center of Biotechnology Information (NCBI) EST database or ftp repository if available. There were 27 citrus cultivars or biotypes with ESTs (Table 1, Additional file 1). In addition to the binomial and common names, the abbreviations for 27 cultivars were designated to facilitate presentation (Table 1, Additional file 1); the binomial names are those used for the accessions in the NCBI database. ESTs were searched for SNPs using the Quality SNP pipeline [8] in each of the 27 cultivars and in three cultivar groups, 12 mandarins (M12), 7 limes/lemons/citron (L7), and all 27 cultivars (C27). The mining results for individual cultivars in the three groups were summed, giving SM12, SL7, and SC27, respectively used to compare with of M12, L7, and C27 (Additional file 1). 'Ridge Pineapple' sweet orange (Citrus sinensis) was selected for SNP validation because the most ESTs and SNPs are from sweet orange and it is a parent to several widely used mapping populations. 
Table 1 Public ESTs in citrus cultivars/biotypes

\begin{tabular}{|c|c|c|c|c|}
\hline No & Binomial names & Common names & Abbreviations & EST numbers \\
\hline 1 & Citrus sinensis & Sweet orange & so & 213,830 \\
\hline 2 & C. clementina & Clementine mandarin & $\mathrm{CM}$ & 122,005 \\
\hline 3 & C. reticulata & Ponkan mandarin & PM & 52,340 \\
\hline 4 & C. unshiu & Satsuma mandarin & SM & 19,072 \\
\hline 5 & C. reshni & Cleopatra mandarin & LM & 5,768 \\
\hline 6 & C. sunki & Hayata mandarin & $\mathrm{HM}$ & 5,216 \\
\hline 7 & C. tamurana & Rixiangxia mandarin & $\mathrm{RM}$ & 358 \\
\hline 8 & C. hassaku & Hassaku mandarin & KM & 154 \\
\hline 9 & C. natsudaidai & Summer orange & UM & 202 \\
\hline 10 & C. reticulata $\times$ C. temple & Orah tangor & OT & 5,823 \\
\hline 11 & C. clementina $\times$ C. reticulata & Fortune tangor & FT & 1,917 \\
\hline 12 & C. nobilis $\times$ C. kinokuni & Kankitsu Chukanbohon Nou 6 Gou tangor & KT & 645 \\
\hline 13 & C. sinensis $\times$ C. reticulata & Amakusa tangor & AT & 160 \\
\hline 14 & C. limonia & Rangpur lime, Mandarin lime & ML & 11,045 \\
\hline 15 & C. Iatifolia & Tahiti lime & $\mathrm{TL}$ & 8,756 \\
\hline 16 & C. aurantifolia & Mexican lime & $\mathrm{KL}$ & 8,219 \\
\hline 17 & C. limettioides & Palestine Sweet lime & SL & 8,188 \\
\hline 18 & C. limon & Lisbon lemon & $\mathrm{LL}$ & 1,505 \\
\hline 19 & C. jambhiri & Rough lemon & $\mathrm{RL}$ & 1,017 \\
\hline 20 & C. medica & Etrog citron & EC & 1,115 \\
\hline 21 & C. aurantium & Sour orange, Bitter orange & BO & 14,584 \\
\hline 22 & C. paradisi & Grapefruit & GF & 8,039 \\
\hline 23 & C. macrophylla & Alemow pepada & AP & 1,929 \\
\hline 24 & C. paradisi $\times$ P. trifoliata & Swingle citrumelo & SC & 7,954 \\
\hline 25 & C. sinensis $\times$ P. trifoliata & Carrizo citrange & $\mathrm{CC}$ & 1,837 \\
\hline 26 & Fortunella margarita & Nagami kumquat & NK & 2,924 \\
\hline \multirow[t]{4}{*}{27} & Poncirus trifoliata & Trifoliate orange & TO & 62,695 \\
\hline & 2-13 combined & & M12 & 213,660 \\
\hline & 14-20 combined & & L7 & 39,845 \\
\hline & 1-27 combined & & $\mathrm{C} 27$ & 567,297 \\
\hline
\end{tabular}

All ESTs are retrieved from the NCBI repository. Those in bold font are over 8,000 ESTs. All mandarin (No. 2-13) and lime/lemon (No. 14-20) types were listed together. The abbreviation for each cultivar and total was designated to facilitate presentation.

\section{SNP discovery and primer design}

The QualitySNP pipeline was installed and used for SNP discovery, following the program manual and recommended parameters [8]. QualitySNP first identified haplotypes in a contig by re-clustering its ESTs and extracted all nucleotide discrepancies (called potential SNPs, pSNPs) between identified haplotypes in a contig, from which a subset of so-called quality SNPs (qSNPs) was identified based on allele and SNP confidence scores defined in the haplotype-based mining algorithm [8]. These qSNP-containing contigs and 25-mer oligo sequences, along with much other mining information, were saved in separate files for database construction and result summary. The ratios of $\mathrm{qSNP} / \mathrm{pSNP}$ were calculated to indicate the percentage of nucleotide discrepancies (pSNPs) identified as high-qaality SNPs (qSNPs) by the QualitySNP algorithm. Bioinformatics programs included in the pipeline were cross_match in the phred-phrap-consed package $[24,25]$ to remove vectors, CAP3 [26] to assemble ESTs, FASTY [27] to align ESTs to the proteins in the Uniprot database for identification of non-synonymous and synonymous SNPs. BatchPrimer3 [28] was used to design a forward (F), a reverse $(\mathrm{R})$, and a single base extension (SBE) primer flanking each SNP site. The F, R and SBE primers of 96 SNPs from $\mathrm{SO}$ were selected for both sequencing and SBE genotyping validation (Additional file 2). After sorting by the lengths of SBE primers, except the first, the 
other 7 primers of every $8 \mathrm{SBE}$ primers were tailed in the 5 ' end with three groups of non-homologous polynucleotides of different lengths to facilitate future multiplex genotyping application. All the F, R and tailed SBE primers, 96 each, were synthesized by Eurofins MWG Operon (Huntsville, Al) in a 96-well plate, respectively, where every three primers of each SNP were placed in the same well of the three different plates and stored in $\mathrm{dd}_{2} \mathrm{O}$ at $10 \mu \mathrm{M}$. The format facilitated easy primer positioning and channel pipetting during the genotyping and sequencing preparation.

\section{SNP 25-nucleotide sequence blast}

All 25-nucleotide oligo sequences (SNP in the middle nucleotide) generated from every citrus genotype by QualitySNP were combined together and used to align to the haploid Clementine reference genome (version 1.0; phytozome.org and citrusgenomedb.org) using BLASTN [29] and a cut-off e-value of 6e-004 (0.0006). Each query sequence (25-mer oligo) against the subject scaffolds would yield either of the following BLASTN outputs, "no hits found", 1 hit on 1 scaffold with 1 alignment, or any other cases (i.e., 1 hit on 1 scaffold with 2+ alignments at different positions or $2+$ hits on different scaffolds with $1+$ alignment each hit). At the preset e value, only alignments with $84 \%$ identities and higher (in other words, only 6 types of alignment hits: $25 / 25,24 / 25,24 / 24,23 / 23,22 / 22$, and 21/21), were saved in the BLASTN output file. The information in the output file, including the scaffold, position, strand, e value, score, alignment identities of each hit, and hit status, was parsed into an EXCEL file to summarize SNP alignment status and to calculate distribution on the Clementine reference genome scaffolds. The information was also used as additional criteria for categorization of SNPs and selection of desired core sets.

\section{SNP validation by sequencing and SNaPshot genotyping assay}

BigDye Terminator V3.1 Cycle Sequencing Kit and SNaPshot Multiplex Kit (Applied Biosystems, Foster City, CA) were used to validate SNPs, following the manufacturer's protocols with some modifications in reaction volumes and/or quantity of proprietary reagents. 96-well plates were used for PCR, enzymatic incubation, and denaturation on iCycler (Bio-Rad, Hercules, CA) and/or GeneAmp PCR System 9700 (Applied Biosystems, Foster City, CA), and for genotyping and sequencing on 3130xl Genetic Analyzer (Applied Biosystems, Foster City, CA). Unless otherwise stated, brief centrifugation up to $1000 \mathrm{rpm}$ in Juan MR 23i was applied after addition of a solution or before implementation of new steps, and all the PCR and enzymatic incubation programs were set to hold at $4^{\circ} \mathrm{C}$ indefinitely at the end until a next procedure.
For both dye terminator sequencing and SNaPshot assays to validate SNPs, template preparation was carried

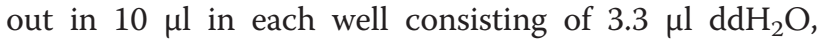
$1.0 \mu \mathrm{l} 10 \mathrm{x}$ dNTPs $(2 \mathrm{mM}), 2.0 \mu \mathrm{l} 5 \mathrm{x}$ colorless GoTaq Flexi buffer, $0.8 \mu \mathrm{l} 25 \mathrm{mM} \mathrm{MgCl} 2,0.4 \mu \mathrm{F}$ and R primers each, $0.1 \mu \mathrm{l} \mathrm{GoTaq} \mathrm{Flexi} \mathrm{(5} \mathrm{units} \mathrm{per} \mu \mathrm{l}$ Promega, Madison, $\mathrm{WI})$, and $2 \mu \mathrm{l}$ genomic DNA (10 $\mathrm{ng} / \mu \mathrm{l})$. The touch-down PCR program started from an initial denaturation at $94^{\circ} \mathrm{C}$ for $3 \mathrm{~min}$, followed by $10 \mathrm{cycles}$ of $93^{\circ} \mathrm{C}$ for $30 \mathrm{sec}, 56^{\circ} \mathrm{C}$ for $45 \mathrm{sec}$ (decreasing $0.5^{\circ} \mathrm{C}$ each annealing step), $72^{\circ} \mathrm{C}$ for $45 \mathrm{sec}$, and 30 continuing cycles with $51^{\circ} \mathrm{C}$ at the annealing step, plus a final elongation at $72^{\circ} \mathrm{C}$ for $15 \mathrm{~min}$. Removal of primers and unused dNTPs was performed by addition of $1 \mu \mathrm{l}$ of ExoISAP-IT (Affymetrix, Santa Clara, CA) into each well of the plate, and incubation at $37^{\circ} \mathrm{C}$ for $60 \mathrm{~min}$ and $75^{\circ} \mathrm{C}$ for $15 \mathrm{~min}$.

Sequencing reactions for SNP validation were prepared in $10 \mu \mathrm{l}$ in each well of a new plate including $2 \mu \mathrm{l}$ $5 \mathrm{x}$ sequencing buffer, $2 \mu \mathrm{l}$ ready reaction premix in the sequencing kit, $1 \mu \mathrm{l} 10 \mu \mathrm{M}$ SNP F primer, and $5 \mu \mathrm{l}$ ExoSAP-IT treated PCR product, started at $95^{\circ} \mathrm{C}$ for $1 \mathrm{~min}$, followed by 25 thermal cycles of $95^{\circ} \mathrm{C}$ for $10 \mathrm{sec}$, $50^{\circ} \mathrm{C}$ for $5 \mathrm{sec}$, and $60^{\circ} \mathrm{C}$ for $4 \mathrm{~min}$. Following the manufacturer's instructions, ethanol/EDTA/sodium acetate precipitation was used to purify the sequencing product in the plate, which was subsequently air dried, then

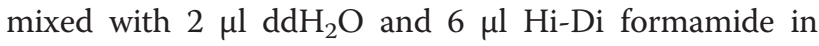
each well, denatured, and loaded to the genetic analyzer to sequence. The sequence files generated were analyzed by Sequencing Analysis software (Applied Biosystems, Foster City, CA) to generate sequences and electropherograms, in which a validated SNP was confirmed by correct alignment of SBE primer sequence into the corresponding sequences and visualization of two different overlapped nucleotide peaks at the nucleotide site in the electropherograms.

The SBE reaction for SNaPshot assays was prepared in $5 \mu \mathrm{l}$ in each well in a new plate including $0.5 \mu \mathrm{l}$ ready reaction premix in the SNaPshot kit, $1 \mu \mathrm{l} \mathrm{SBE} 10 \mu \mathrm{M}$ primer, and $3.5 \mu \mathrm{l}$ ExoSAP-IT treated PCR product, and repeated in 25 thermal cycles of $95^{\circ} \mathrm{C}$ for $10 \mathrm{sec}, 50^{\circ} \mathrm{C}$ for $5 \mathrm{sec}$, and $60^{\circ} \mathrm{C}$ for $30 \mathrm{sec}$. Removal of unincorporated dye-labeled ddNTPs was completed by addition of

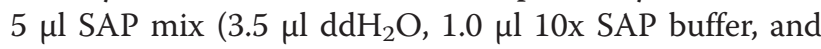
$0.5 \mu \mathrm{l} 1 \mathrm{u} / \mu \mathrm{l} \mathrm{SAP}$ ) into the SBE reaction mix, and incubation at $37^{\circ} \mathrm{C}$ for $60 \mathrm{~min}$ and $75^{\circ} \mathrm{C}$ for $15 \mathrm{~min}$. Genotyping was performed using $8 \mu \mathrm{lmix}$ in each well of a new plate consisting of $1 \mu \mathrm{l}$ SAP treated SBE product, $0.25 \mu \mathrm{l}$ Gene Scan $120 \mathrm{LIZ}$ size standard, and $6.75 \mu \mathrm{l} \mathrm{Hi-Di} \mathrm{formamide,}$ which was denatured at $95^{\circ} \mathrm{C} 3 \mathrm{~min}$ then immediately moved on ice for at least $2 \mathrm{~min}$. The SNaPshot files were used to score SNPs by GeneMarker (SoftGenetics, State College, PA) in which a validated SNP consisted of two different nucleotides. 


\section{Results}

\section{Haplotype-based EST-SNPs in citrus cultivars}

Haplotype-based SNPs were mined from ESTs of the 27 citrus cultivars and 3 groups (M12 - 12 mandarins, L7 7 limes/lemons, and C27 - all 27 combined) using the QualitySNP pipeline and summarized in detail (Additional file 1). In summary (SC27 - the last column in Additional file 1), a total of 25,417 qSNPs (Additional file 2) were identified from ESTs of the 27 cultivars mined separately. These are attributed to heterozygosity within cultivars at SNP loci. There were only 2805 SNPs duplicated according to comparison of all the 25 -mer oligo sequences. The percentages of the 7 SNP types were similar among most citrus cultivars with each type of quality SNPs found. Among the 25,417 qSNPs summed from the 27 citrus cultivars, 15,010 (59.1\%) were transitions (AG and CT), 9,114 (35.9\%) transversions (AC, GT, CG, and AT), and 1,293 $(5.0 \%)$ insertion/deletion events (indels). On average, there were 2.4 SNPs per contig and one SNP every 1,064 bp in all of the SNP-containing contig sequences (Figure 1; Additional file 1).

For individual cultivars, their numbers of ESTs were different, so consequentially were their quality SNPs and other related numbers. For example, in SO, 213,830 ESTs yielded 7,404 contigs of $>=4$ ESTs. Of these, 4,228 contigs contained 43,655 potential SNPs and 3,327 contained qSNPs. The total number of qSNPs was 11,182 . In other words, there was only one haplotype detected in 3,176 contigs $(7,404$ minus 4,228$)$ and no quality SNP identified in the additional 1,001 contigs $(4,428$ minus 3,327 ) with potential SNPs. There were 3.4 quality SNPs per contig and one quality SNP per $723 \mathrm{bp}$ in the contigs on average. Of these 11,182 qSNPs, 6,822 (61.0\%) were transitions (AG and CT type), 3,879 (34.7\%) transversions (AC, GT, CG, and AT type), and 481 (4.3\%) insertion/deletion (Indels); and 2,619 (23.4\%) were nsSNPs and 4,038 (36.1\%) were sSNPs. The absolute numbers of quality SNPs were not comparable due to varying numbers of ESTs among citrus cultivars, but the number of potential and quality SNPs from each cultivar were strongly correlated with its number of ESTs; more ESTs yielded more usable contigs $(>=4$ ESTs) available for SNP mining, as well as more quality SNPs (Additional file 1). Given the large differences in the numbers of ESTs available among the various cultivars, it is more interesting to compare SNP frequencies, rates, and ratios among cultivars with substantial EST numbers and distinct genetic backgrounds, and differences between the mining results of the three grouped ESTs (M12, L7, and C27) and the three sums/averages (SM12, SL7, and SC27) of separately mined counterpart individuals. These comparisons will be elaborated hereafter.

\section{Haplotypes detected in contigs with SNPs}

One important feature of QualitySNP is to re-cluster ESTs in a contig to reconstruct and determine the haplotypes in that contig, from which only single nucleotide discrepancies between any two defined haplotypes (allelic sequences) are considered as potential SNPs for further quality and confidence interrogation. Only those potential SNPs passing confidence scores are identified as quality SNPs. In Additional file 1, all the haplotypes

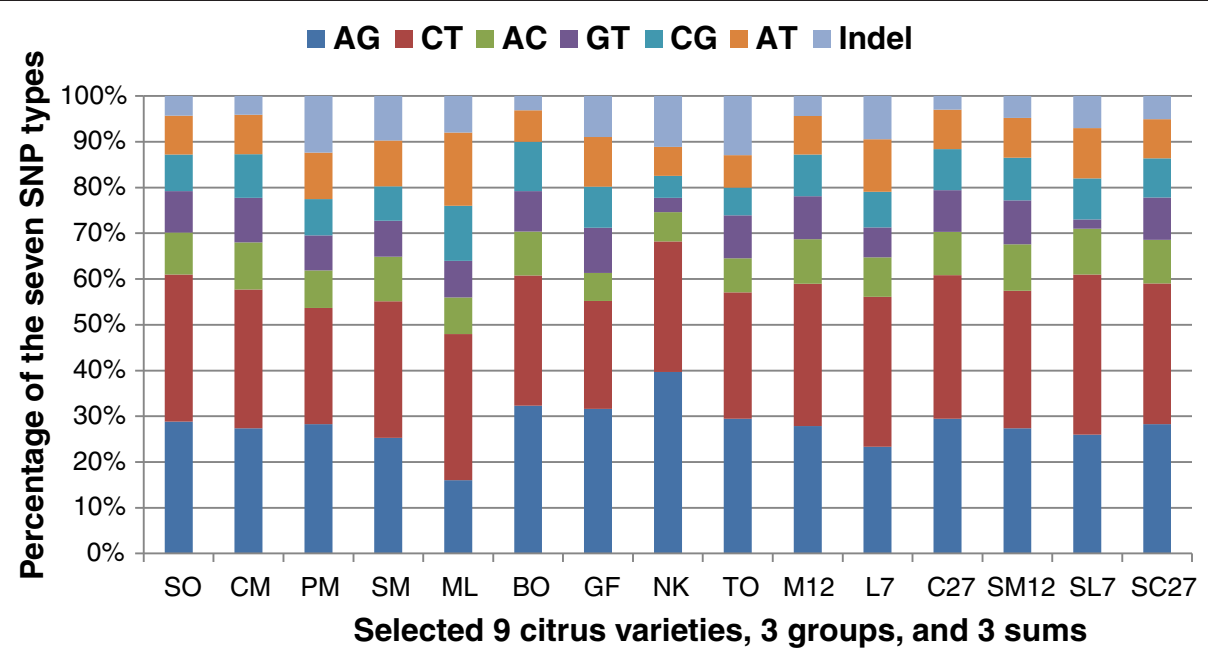

Figure 1 Percentages of the 7 SNP types, AG, CT, AC, GT, CG, AT, and indel, discovered from citrus ESTs. Presented here are 9 selected citrus cultivars, 3 groups, and 3 sums. SO, Sweet orange; CM, Clementine mandarin; PM, Ponkan mandarin; SM, Satsuma mandarin; ML, Rangpur lime; BO, Sour orange; GF, Grapefruit; NK, Nagami kumquat; TO, Trifoliate orange; M12, SNPs from ESTs combined from 12 mandarins (2-13 in Table 1), L7, SNPs from ESTs combined from 7 limes / lemons (14-20 in Table 1); C27, SNPs from all ESTs combined (1-27 in Table 1); SM12, SL7 and SC27, the respective sum of the 12 mandarins, 7 limes/lemons, and all 27 cultivars. On the average of the 27 cultivars (SC27), transitions (AG and $\mathrm{CT}$ ) account for 59.1\%, transversions (AC, GT, CG, and AT) for 35.9\%, and insertion/deletions (indels) for 5.0\%. 
detected in the SNP-containing contigs from all the 27 citrus cultivars are included. Theoretically, there should be only a maximum of 2 haplotypes detected in a diploid genome. As expected, a vast majority of SNP-containing contigs consisted of two haplotypes, but the percentages of 2 haplotypes varied in a wide range in these citrus cultivars (Figure 2, Additional file 1). Among the highest were ML (92\%), SC (84\%), and GF (76\%), and among the lowest PM (38\%), KL (42\%), and CM (48\%). The variation likely results from the genetic makeup of the "cultivar" used to generate the ESTs. For example, ESTs for SO came from navel oranges, blood oranges, and others named $C$. sinensis, rather than a single genotype. In contrast, other "cultivars" are likely single clones. It was also evident as expected that much lower percentages of 2 haplotypes were found in three combined EST datasets (M12, 44\%; L7, 70\%; and C27, 34\%) due to introduction of more haplotypes from different types of citrus cultivars, compared to their counterpart averages of each group (SM12, 48\%; SL7, 74\%; and SC27, 53\%). As a consequence, more qSNPs in higher qSNPs/pSNPs and qSNPs/ESTs ratios were found in the three grouped EST datasets (M12, L7, and C27), compared to their counterparts (SM12, SL7, and SC27) summed from the individually mined cultivar EST results, but the ratio of contigs with qSNPs and contigs used was the opposite (Figure 3, Additional file 1). The frequency of qSNPs is much higher in the pooled data for the three groups (M12, L7 and C27) than in the summed data for individual cultivars. This is because the group values include polymorphism among homozygous accessions as well as heterozygosity within cultivars, while the summed data include only SNPs due to heterozygosity. In other words, the nucleotide at such a SNP is very likely homozygous within a genotype, making it useless in genetic linkage mapping of that genotype.

\section{Alignment and distribution on the Clementine reference genome}

A total of 25,417 25-mer sequences (query sequence, Additional file 2) with quality SNPs from all the 27 citrus cultivars were used to align to the Clementine reference scaffolds (subject sequence) using BLASTN at a cut-off e-value of 6e-004 (Table 2). 2,947 sequences had "no hits found" and 22,470 one or more hits. Of the 22,470 SNPs with hits, 19,943 had only 1 scaffold hit with only 1 alignment on the scaffold, 1,571 had 1 scaffold hit but $>=2$ alignments on the scaffold ( 3 alignments per scaffold hit on average), and 956 had $>=2$ scaffold hits ( $\sim 3$ hits per oligo on average) with 1 or more alignments on each of the scaffolds ( 7 alignments per scaffold hit or $\sim 20$ alignments per oligo on average). It suggested the 19,943 25-mer oligo sequences appear to be unique in the genome, and the remaining 2,527 25mer sequences may have duplicated or similar sequences with at least $84 \%$ identities at different locations in the genome. There was one extreme case that one 25-mer sequence from trifoliate orange yielded 29 scaffold hits and 2,162 alignments on all the scaffolds, the highest numbers of all.

Taking these multiple scaffold hits and alignments into account, the total number of scaffold hits was 24,293 with a total of 43,668 alignments on the scaffolds. Most had $100 \%(25 / 25)$ or $96 \%(24 / 25)$ nucleotide identities to

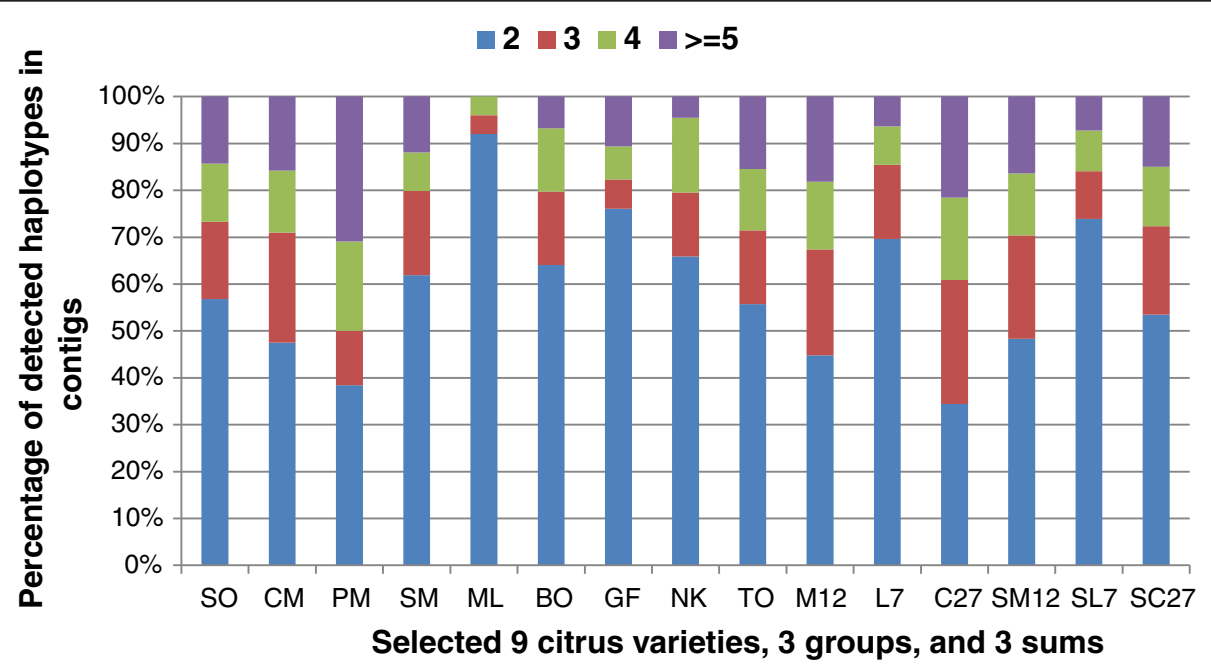

Figure 2 Percentages of detected haplotype numbers $(\mathbf{2}, \mathbf{3}, \mathbf{4}$, and $>=\mathbf{5}$ ) in contigs (>=4 ESTs) with potential SNPs. Presented here are 9 selected citrus cultivars, 3 groups, and 3 sums. SO, Sweet orange; CM, Clementine mandarin; PM, Ponkan mandarin; SM, Satsuma mandarin; ML, Rangpur lime; BO, Sour orange; GF, Grapefruit; NK, Nagami kumquat; TO, Trifoliate orange; M12, SNPs from ESTs combined from 12 mandarins (2-13 in Table 1), L7, SNPs from ESTs combined from 7 limes/lemons (14-20 in Table 1); C27, SNPs from all ESTs combined (1-27 in Table 1); SM12, SL7 and SC27, the respective sum of the 12 mandarins, 7 limes/lemons, and all 27 cultivars. 


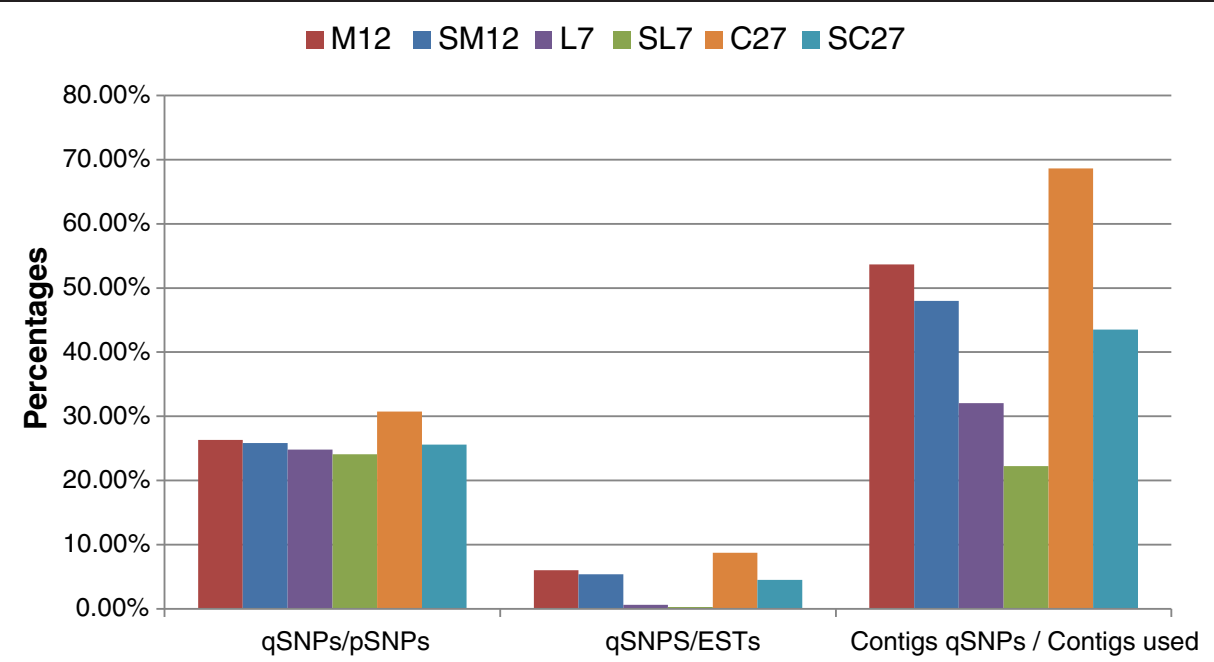

Figure 3 Comparisons between M12 vs. SM12, L7 vs. SL7, and C27 vs. SC27, respectively in three ratios. There are three ratios presented as percentage, qSNPs, the number of quality SNPs; PSNPs, the number of potential SNPs; ESTs, the number of ESTs; contigs qSNPs, the number of contigs with qSNPs; contigs used, the number of contigs with >=4 ESTs. M12, L7 and C27 are mined from grouped ESTs from the corresponding cultivars, and SM12, SL7, and SC27 summed from individually mined cultivars used in the grouped counterparts, respectively.

those on the reference genome, accounting for $93 \%$ of all the alignments. Almost all the nucleotide discrepancies in the 24/25 alignments were at the SNP sites, which is an encouraging in silico validation of these SNPs. Of the total 24,293 scaffold hits, 23,955 were on main scaffolds 1 to $9(2,122,2,804,4,159,2,813,3,045$, $2,501,1,861,2,308$, and 2,342, respectively), accounting for $98.6 \%$ of the total. The remaining 338 were on 87 small scaffolds. Figure 4 showed the distribution of SNPs with all and unique hits from $\mathrm{SO}, \mathrm{TO}$, and $\mathrm{CM}$ on scaffold_1 of the haploid Clementine genome (similar figures on scaffold_2 are in Additional file 3). According to the aligned SNP counts on each $500 \mathrm{~kb}$, there were some featured regions (intervals in Figure 4). For example, in SO many fewer unique hits were found in the middle region, compared to those in two arm regions. Relatively even distribution was observed in CM, with exceptions at Interval 5 with overwhelming duplicated hits of certain SNPs (similar to the same region in SO). There were very limited unique SNPs aligned at Interval 20-27 of all the three cultivars, suggesting the region may contain the centromere, usually characterized by fewer genes. These results, combined with other criteria, should greatly facilitate selection of well-distributed core sets of SNPs across citrus genomes for different genotyping applications and genetic studies.

\section{SNP validation by sequencing and SNaPshot genotyping assay}

Of the 96 randomly selected sweet orange SNPs, 68 were validated by sequencing and 74 by $\mathrm{SNaPshot}$ in sweet orange (Additional file 4). There were 61 validated by both assays and the remainder validated by only one assay. In other words, 7 were validated by only sequencing but failed in SNaPshot, and 13 by only SNaPshot but failed in sequencing. Therefore, a total of 81 SNPs $(84 \%)$ were validated by at least one of the two assays. The high rate $(84 \%)$ of validated SNPs was consistent with $93 \%$ alignments onto the reference genome with $100 \%(25 / 25)$ or $96 \%(24 / 25)$ identities (Table 2$)$, indicating

Table 2 BLASTN results of 25,417 25-mer oligo sequences

\begin{tabular}{lrrrrrrrrr}
\hline & $\mathbf{2 5}$-mers & Hits & Alns & $\mathbf{2 5 / 2 5}$ & $\mathbf{2 4 / 2 5}$ & $\mathbf{2 4 / 2 4}$ & $\mathbf{2 3 / 2 3}$ & $\mathbf{2 2 / 2 2}$ & $\mathbf{2 1 / 2 1}$ \\
\hline No hits found & 2,947 & & & & & & & & \\
1 hit $(1$ aln) & 19,943 & 19,943 & 19,943 & 10,926 & 8,555 & 127 & 116 & 112 & 107 \\
1 hit $(2+$ aln) & 1,571 & 1,571 & 4,614 & 2,152 & 2,026 & 161 & 73 & 78 & 124 \\
$2+$ hits $(1+$ aln each hit) & 956 & 2,779 & 19,111 & 7,923 & 9,014 & 389 & 353 & 715 & 717 \\
Total & 25,417 & 24,293 & 43,668 & 21,001 & 19,595 & 677 & 542 & 905 & 948 \\
\hline
\end{tabular}

The Clementine reference genome was used as the BLAST database. All the oligo sequences were listed in Additional file 2. Aln - Alignment(s); 1 hit (1 aln) - hit only 1 scaffold with 1 alignment; 1 hit $(2+$ aln $)$ - hit on only 1 scaffold but with 2 or more alignments, and $2+$ hits $(1+$ aln $)-$ hit on 2 and more scaffolds with one or more alignment to each scaffold. 

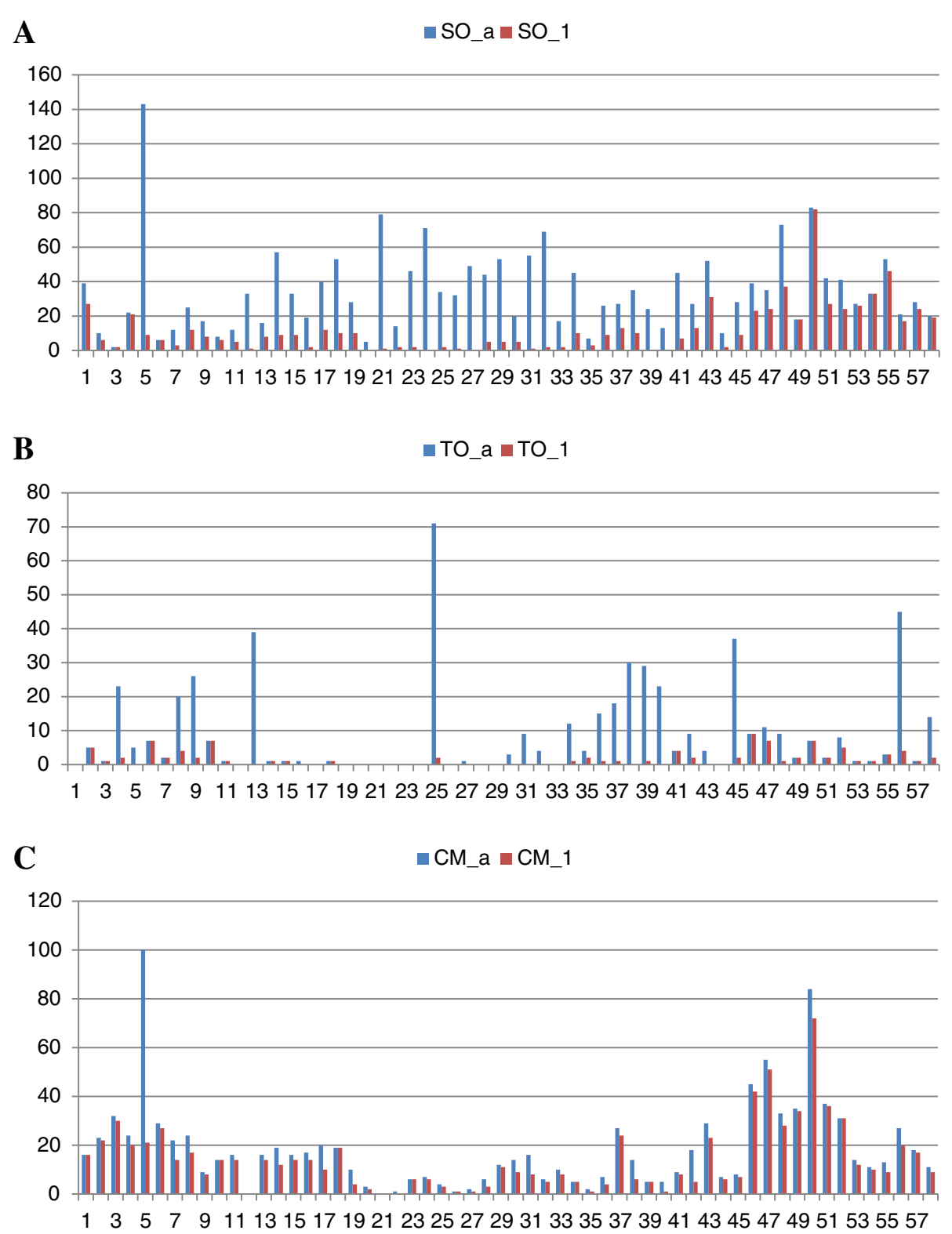

Figure 4 SNP distribution on the Clementine reference genome, using Scaffold_1 as an example. Each interval of the $x$-axis represented $500 \mathrm{~kb}$ of the scaffold, and the $\mathrm{y}$-axis represented the number of SNPs in each $500 \mathrm{~kb}$ on the scaffold. SO - sweet orange (A); TO - trifoliate orange (B); CM - Clementine mandarin (C); "_a" - counts of all alignments generated by all SNPs; "_1" - counts of SNPs of only 1 unique hit/alignment in the genome. Differences between the "_a" and "_1" numbers are observed in several regions of each cultivar.

that QualitySNP, a haplotype-based SNP mining algorithm and pipeline, is a very reliable tool to identify true EST SNPs, and it can effectively minimize the false discovery rate even without quality files.

\section{Discussion}

Estimation of heterozygosity of different citrus genomes by haplotype-based SNPs

Many naturally evolved genomes are heterozygous, and the heterozygosity level may be evaluated by the rate of allelic nucleotide variations between the two haplotypes [30]. SNPs, the most abundant polymorphisms in genomes, likely are the most appropriate index for the heterozygosity levels of genetically/taxonomically related genomes [19,21,22]. Given the different numbers and rates of haplotype-based SNPs discovered from these citrus individuals with substantial numbers of ESTs (for example more than 5,000, Additional file 1), the ratios of qSNPs/ESTs in most of them appeared reflective of their heterozygous status and genetic background. These 
hybrid derivatives had much higher qSNPs/ESTs ratio, while the other believed "pure" species had lower ratios. For example, some proven natural hybrid cultivars, such as $\mathrm{SO}, \mathrm{CM}$, and recent hybrids such as SC, were among the higher qSNPs/ESTs ratios (SO - 5.23\%, CM - 8.31\%, and $\mathrm{SC}-7.76 \%$ ). Other presumed true species, including $\mathrm{PM}$, fell in the lower qSNP/ESTs ratios (PM - 0.60\%). The number of needed ESTs to generate the desired number of SNPs in given citrus genotypes, and vice versa, can be estimated. Such a tendency, along with the ratios and genome heterozygosity, could be strengthened and would be more conclusive if the numbers of ESTs in all the cultivars were close to each other, or at least in a much smaller range.

\section{SNP discovery and validation rates}

SNP mining is no longer a bottleneck because computational capacity and sequence data are exponentially increasing, and more SNP mining pipelines have become available in recent years [7,8,12-15,31]. Hundreds of thousands of SNPs can be easily mined out of EST or genomic sequences. Inclusion of false SNPs in genotyping certainly is wasteful; therefore, maximizing the true SNP rate (minimizing the false rate) is the most important consideration or requirement for a SNP mining algorithm because any validation approach can only validate these true SNPs, but not false ones $[8,13]$. We found that 93\% of SNPs identified by the QualitySNP pipeline were aligned onto the reference genome at $25 / 25$ or $24 / 25$ identities, and $81 \%$ of randomly selected sweet orange SNPs were validated by sequencing and SNaPshot genotyping. It was undetermined whether the others not aligned at the two identity rates, and not validated by sequencing and/or genotyping, were true or false SNPs. For example, those failing in sequencing validation might be due to SBE primer sequences not being found (likely an intron in the region), or sequencing failures caused by primers of low quality or in a variable region, or no nucleotide discrepancies at the sites. It was unclear how these SNPs failed in SNaPshot validation; it is speculated some of these SBE primers might be incorrectly positioned, i.e., the singly extended nucleotides may not have been exactly at the SNP sites. There were a few such cases identified (Chen et al. unpublished data); very likely due to the differences between these consensus contigs and the original haplotype sequences. On the other hand, only 2 haplotypes may exist in a diploid genome. If SNPs were from the contigs with more than 2 haplotypes, such cases could result from either ESTs mixed from diverse genotypes in the same species or highly identical paralogs assembled into the contigs. Paralogous genes, resulting from genomic duplication and evolving into different functions, are very common in many genomes and remain almost identical in their conserved regions. ESTs from different paralogous genes, if assembled into a same unigene, could yield false SNPs that are non-allelic and useless.

\section{Criteria for selection of citrus core SNP sets}

In most cases the discovered SNPs could easily reach a number so large that only a small portion of them, designated core SNP set, are selected and used in genotyping to meet the restraints in available budget, desired platform, applications, and other factors [3,11,32-34]. These core sets of different numbers (e.g. 384, 1536, or other numbers) are either required by certain SNP genotyping platforms or optimized for particular applications [35-38]. It may be a daunting job, but it is necessary to establish workable criteria to select any core set of different numbers of SNPs. Based on this complete mining and validation process, several attributes of SNPs can be very useful and distinguishing to refine these core sets of different numbers. SNP oligo alignment uniqueness, identity percentage, and distribution in the reference genome, co-existence across different genomes, along with SNP types (nsSNP vs. sSNP, and transition vs. transversion vs. indel) and numbers per gene, should be the main criteria for selection of citrus core SNP sets. As pointed out, some extra haplotypes might result from paralogs across different genome regions. In that case, the resulting SNPs would not be allelic or useful. Whether they mostly were those SNPs that had multiple scaffold hits and alignments remains unclear pending further investigation. Those SNPs from either circumstance should be excluded or at least deprioritized for use in genotyping. Selection of SNPs for genotyping could be difficult when different attributes of SNPs and genotyping platforms are considered. A tool based on these attributes is being developed to achieve the automatic selection of core SNP sets for targeted applications/platforms [35,36] and to allow geneticists and molecular breeders to be able to select and use certain core SNPs of interest from among the thousands discovered $[37,38]$. All the SNPs (Additional file 2) identified in this work are being added to a citrus genome database (citrusgenomedb.org). Very recently after this study, another draft genome of sweet orange was reported, yielding 1.06 million genome-wide SNPs, about 3.6 SNPs/kb, which could be an additional valuable resource in SNP applications [39].

\section{Conclusions}

High-quality SNPs in public ESTs from different citrus genotypes were detected by the QualitySNP pipeline and compared to estimate the heterozygosity of each genome. All the short SNP oligo sequences were also aligned with the Clementine citrus genome to determine their distribution and uniqueness in the genome and for 
in silico validation. Selected SNPs were also validated by $\mathrm{SNaPshot}$ and sequencing.

\section{Additional files}

Additional file 1: Table S1. Summary of citrus EST SNPS. It includes mining results from 27 individual varieties with their index number, binomial name, common name, and abbreviation, 3 grouped ESTs - M12, 12 mandarins (2-13); L7, 7 limes/lemons (14-20); C27, all 27 citrus varieties (1-27); and three summed/averaged results, SM12, SL7 and SC27, respectively from the 12 individually mined mandarins, 7 limes/lemons, and all 27 varieties, which were used for comparison to M12, L7, and C27.

Additional file 2: Table S2. 25417 25-mer sequences of SNPs and forward, reverse, single base extension (SBE) primer, and SBE 5'-tail sequences for 96 SNPs selected from sweet orange.

Additional file 3: Figure S1. SNP distribution on the Clementine reference genome Scaffold_2. Each interval of the x-axis represented $500 \mathrm{~kb}$ of the scaffold, and the $y$-axis represented the number of SNPs in each $500 \mathrm{~kb}$ on the scaffold. SO - sweet orange (A); TO - trifoliate orange (B); CM - Clementine mandarin (C); " $a$ " - counts of all alignments generated by all SNPs; "_1" - counts of SNPs of only 1 unique hit/alignment in the genome. Differences between the "_ $a$ " and "_1" numbers were observed in several regions of each cultivar.

Additional file 4: Figure S2. SNapShot chromatograph of a SNP validated by the assay, generated by GeneMarker (SoftGenetics, State College, PA). The $y$-axis represents the intensity of, and $x$-axis the approximate length of, the fluorescently-labeled SBE products ending with A and G.

\section{Competing interests}

The authors declare that they have no competing interests.

\section{Authors' contributions}

CC conceived the study, performed bioinformatics analysis and comparison, and wrote the manuscript, and FG critically read and revised the manuscript. All the authors read and approved the final manuscript.

\section{Acknowledgements}

We thank Dr. Harm Nijveen for contributing his valuable time to fix a bug in one C program in the QualitySNP pipeline and Dr. Frank You to modify some code in the BatchPrimer3 script to meet some primer output requests. The work was partially supported by grants from the Citrus Research and Development Foundation (\#67, \#71), on behalf of the Florida citrus growers.

Received: 20 February 2013 Accepted: 22 October 2013

Published: 1 November 2013

\section{References}

1. Brookes AJ: The essence of SNPs. Gene 1999, 234(2):177-186

2. Dawson E: New collaborations make pharmacogenomics a SNP. Mol Med Today 1999, 5(7):280.

3. Rickert AM, Kim JH, Meyer S, Nagel A, Ballvora A, Oefner PJ, Gebhardt C First-generation SNP/InDel markers tagging loci for pathogen resistance in the potato genome. Plant Biotechnol J 2003, 1(6):399-410.

4. Han Y, Chagne D, Gasic K, Rikkerink EH, Beever JE, Gardiner SE, Korban SS: BAC-end sequence-based SNPs and Bin mapping for rapid integration of physical and genetic maps in apple. Genomics 2009, 93(3):282-288.

5. Garg K, Green P, Nickerson DA: Identification of candidate coding region single nucleotide polymorphisms in 165 human genes using assembled expressed sequence tags. Genome Res 1999, 9(11):1087-1092.

6. Picoult-Newberg L, Ideker TE, Pohl MG, Taylor SL, Donaldson MA, Nickerson DA, Boyce-Jacino M: Mining SNPs from EST databases. Genome Res 1999, 9(2):167-174.

7. Batley J, Barker G, O'Sullivan H, Edwards KJ, Edwards D: Mining for single nucleotide polymorphisms and insertions/deletions in maize expressed sequence tag data. Plant Physiol 2003, 132(1):84-91.

8. Tang J, Vosman B, Voorrips RE, van der Linden CG, Leunissen JA QualitySNP: a pipeline for detecting single nucleotide polymorphisms and insertions/deletions in EST data from diploid and polyploid species. BMC Bioinformatics 2006, 7:438.

9. Kim H, Schmidt CJ, Decker KS, Emara MG: A double-screening method to identify reliable candidate non-synonymous SNPs from chicken EST data. Anim Genet 2003, 34(4):249-254.

10. Matukumalli LK, Grefenstette JJ, Hyten DL, Choi IY, Cregan PB, Van Tassell CP: Application of machine learning in SNP discovery. BMC Bioinformatics 2006, 7:4.

11. Mooney S: Bioinformatics approaches and resources for single nucleotide polymorphism functional analysis. Brief Bioinform 2005, 6(1):44-56.

12. Nickerson DA, Tobe VO, Taylor SL: PolyPhred: automating the detection and genotyping of single nucleotide substitutions using fluorescencebased resequencing. Nucleic Acids Res 1997, 25(14):2745-2751.

13. Marth GT, Korf I, Yandell MD, Yeh RT, Gu Z, Zakeri H, Stitziel NO, Hillier L, Kwok PY, Gish WR: A general approach to single-nucleotide polymorphism discovery. Nat Genet 1999, 23(4):452-456.

14. Montgomery KT, lartchouck O, Li L, Loomis S, Obourn V, Kucherlapati R: PolyPhred analysis software for mutation detection from fluorescencebased sequence data. Curr Protoc Hum Genet 2008, Chapter 7:Unit 7.16.1-21.

15. Barker G, Batley J, O'Sullivan H, Edwards KJ, Edwards D: Redundancy based detection of sequence polymorphisms in expressed sequence tag data using autoSNP. Bioinformatics 2003, 19(3):421-422.

16. Gmitter F Jr, Chen C, Machado M, Souza A, Ollitrault P, Froehlicher Y, Shimizu T: Citrus genomics. Tree Genet Genomes 2012, 8(3):611-626.

17. Federici C, Fang D, Scora R, Roose M: Phylogenetic relationships within the genus Citrus (Rutaceae) and related genera as revealed by RFLP and RAPD analysis. Theor Appl Genet 1998, 96:812-822.

18. Nicolosi E, Deng Z, Gentile A, La Malfa S, Continella G, Tribulato E: Citrus phylogeny and genetic origin of important species as investigated by molecular markers. Theor Appl Genet 2000, 100:1155-1166.

19. Chen C, Zhou P, Choi YA, Huang S, Gmitter FG Jr: Mining and characterizing microsatellites from citrus ESTs. Theor Appl Genet 2006, 112(7):1248-1257.

20. Chen C, Bowman K, Choi Y, Dang P, Rao M, Huang S, Soneji J, McCollum TG, Gmitter F Jr: EST-SSR genetic maps for Citrus sinensis and Poncirus trifoliata. Tree Genet Genomes 2008, 4(1):1-10.

21. Ollitrault $P$, Terol J, Chen C, Federici CT, Lotfy S, Hippolyte I, Ollitrault F, Berard A, Chauveau A, Cuenca J, Costantino G, Kacar Y, Mu L, Garcia-Lor A, Froelicher Y, Aleza P, Boland A, Billot C, Navarro L, Luro F, Roose ML, Gmitter $\mathrm{FG}$, Talon M, Brunel D: A reference genetic map of $C$. clementina hort. ex Tan.; citrus evolution inferences from comparative mapping. BMC Genomics 2012, 13:593-2164. 13-593.

22. Gmitter FG Jr: The haploid mandarin and diploid sweet orange genome sequences. Plant \& Anim Genomes XIX Conference 2011:W146 [abstract].

23. Chen C, Cancalon P, Haun C, Gmitter F: Characterization of furanocoumarin profile and inheritance toward selection of low furanocoumarin seedless grapefruit cultivars. J Am Soc Hort Sci 2011, 136:358-363.

24. Gordon D: Viewing and editing assembled sequences using Consed. Curr Protoc Bioinformatics 2003, Chapter 11:Unit11.2.1-11.

25. de la Bastide M, McCombie WR: Assembling genomic DNA sequences with PHRAP. Curr Protoc Bioinformatics 2007, Chapter 11:Unit11.4.1-7.

26. Huang X, Madan A: CAP3: A DNA sequence assembly program. Genome Res 1999, 9(9):868-877.

27. Pearson WR, Wood T, Zhang Z, Miller W: Comparison of DNA sequences with protein sequences. Genomics 1997, 46(1):24-36.

28. You FM, Huo N, Gu YQ, Luo MC, Ma Y, Hane D, Lazo GR, Dvorak J, Anderson OD: BatchPrimer3: a high throughput web application for PCR and sequencing primer design. BMC Bioinformatics 2008, 9:253.

29. Altschul SF, Madden TL, Schaffer AA, Zhang J, Zhang Z, Miller W, Lipman DJ: Gapped BLAST and PSI-BLAST: a new generation of protein database search programs. Nucleic Acids Res 1997, 25(17):3389-3402.

30. Moroldo M, Paillard S, Marconi R, Fabrice L, Canaguier A, Cruaud C, De Berardinis V, Guichard C, Brunaud V, Le Clainche I, Scalabrin S, Testolin R, Di Gaspero G, Morgante M, Adam-Blondon AF: A physical map of the heterozygous grapevine 'Cabernet Sauvignon' allows mapping candidate genes for disease resistance. BMC Plant Biol 2008, 8:66.

31. Clifford RJ, Edmonson MN, Nguyen C, Scherpbier T, Hu Y, Buetow KH: Bioinformatics tools for single nucleotide polymorphism discovery and analysis. Ann N Y Acad Sci 2004, 1020:101-109.

32. Rahmann S: Fast large scale oligonucleotide selection using the longest common factor approach. J Bioinform Comput Biol 2003, 1(2):343-361 
33. Jena KK, Jeung JU, Lee JH, Choi HC, Brar DS: High-resolution mapping of a new brown planthopper $(\mathrm{BPH})$ resistance gene, $\mathrm{Bph} 18(\mathrm{t})$, and markerassisted selection for BPH resistance in rice (Oryza sativa L.). Theor Appl Genet 2006, 112(2):288-297.

34. Shulaev V, Korban SS, Sosinski B, Abbott AG, Aldwinckle HS, Folta KM, lezzoni A, Main D, Arus P, Dandekar AM, Lewers K, Brown SK, Davis TM, Gardiner SE, Potter D, Veilleux RE: Multiple models for Rosaceae genomics. Plant Physiol 2008, 147(3):985-1003.

35. Ahmadi KR, Weale ME, Xue ZY, Soranzo N, Yarnall DP, Briley JD, Maruyama Y, Kobayashi M, Wood NW, Spurr NK, Burns DK, Roses AD, Saunders AM, Goldstein DB: A single-nucleotide polymorphism tagging set for human drug metabolism and transport. Nat Genet 2005, 37(1):84-89.

36. Chagne D, Gasic K, Crowhurst RN, Han Y, Bassett HC, Bowatte DR, Lawrence TJ, Rikkerink EH, Gardiner SE, Korban SS: Development of a set of SNP markers present in expressed genes of the apple. Genomics 2008, 92(5):353-358

37. Harlizius $B$, Lopes MS, Duijvesteijn $N$, van de Goor $L H$, van Haeringen WA, Panneman H, Guimaraes SE, Merks JW, Knol EF: A single nucleotide polymorphism set for paternal identification to reduce the costs of trait recording in commercial pig breeding. J Anim Sci 2011, 89(6):1661-1668.

38. Greenawalt DM, Sieberts SK, Cornelis MC, Girman CJ, Zhong H, Yang X, Guinney J, Qi L, Hu FB: Integrating genetic association, genetics of gene expression, and single nucleotide polymorphism set analysis to identify susceptibility loci for type 2 diabetes mellitus. Am J Epidemiol 2012, 176(5):423-430.

39. Xu Q, Chen LL, Ruan X, Chen D, Zhu A, Chen C, Bertrand D, Jiao WB, Hao BH, Lyon MP, Chen J, Gao S, Xing F, Lan H, Chang JW, Ge X, Lei Y, Hu Q, Miao Y, Wang L, Xiao S, Biswas MK, Zeng W, Guo F, Cao H, Yang X, Xu XW Cheng YJ, Xu J, Liu JH, Luo OJ, Tang Z, Guo WW, Kuang H, Zhang HY, Roose ML, Nagarajan N, Deng XX, Ruan Y: The draft genome of sweet orange (Citrus sinensis). Nat Genet 2013, 45(1):59-66.

doi:10.1186/1471-2164-14-746

Cite this article as: Chen and Gmitter: Mining of haplotype-based expressed sequence tag single nucleotide polymorphisms in citrus. $B M C$ Genomics 2013 14:746.

\section{Submit your next manuscript to BioMed Central and take full advantage of:}

- Convenient online submission

- Thorough peer review

- No space constraints or color figure charges

- Immediate publication on acceptance

- Inclusion in PubMed, CAS, Scopus and Google Scholar

- Research which is freely available for redistribution 